\title{
Ion implantation of CdTe single crystals
}

\author{
Tomasz Wiecek ${ }^{1}$, Volodymir Popovich², Mariusz Bester ${ }^{3}$, Marian Kuzma $^{3}$ \\ ${ }^{1}$ Physics Chair, Rzeszow University of Technology, al. Powstańców Warszawy 12, 35-959 Rzeszow, Poland \\ ${ }^{2}$ Department of Fundamental Technologies, Ivan Franko Drogobych State Pedagogical University, \\ Ivan Franko 24, 82100 Drogobych, Ukraine \\ ${ }^{3}$ Biophysics Chair, Rzeszow University, S. Pigonia 1, 35-959 Rzeszow, Poland
}

\begin{abstract}
Ion implantation is a technique which is widely used in industry for unique modification of metal surface for medical applications. In semiconductor silicon technology ion implantation is also widely used for thin layer electronic or optoelectronic devices production. For other semiconductor materials this technique is still at an early stage. In this paper based on literature data we present the main features of the implantation of CdTe single crystals as well as some of the major problems which are likely to occur when dealing with them. The most unexpected feature is the high resistance of these crystals against the amorphization caused by ion implantation even at high doses $\left(10^{17} 1 / \mathrm{cm}^{2}\right)$. The second property is the disposal of defects much deeper in the sample then it follows from the modeling calculations. The outline of principles of the ion implantation is included in the paper. The data based on RBS measurements and modeling results obtained by using SRIM software were taken into account.
\end{abstract}

\section{Introduction}

Doping of single crystals of CdTe is a troublesome task in AIIBVI devices technology due to the segregation effects, the presence of compensating defects in the crystal and due to disturbing of stoichiometry [1].There are three main methods of doping of CdTe crystals : the first being the addition of species during growth, the second one reckoning on the diffusion of dopantss using annealing, and the third one which is the ion implantation. The two first methods are extensively experimentally investigated for wide amount of dopant elements ( see Chapter B5 in [1]). Contrarily, the ion implantation has been studied for two dopants only : As and $\mathrm{Bi}$. The aim of such studies was type conversion for $\mathrm{p}-\mathrm{n}$ junction formation. Ion implantation is broadly applied in semiconductor, and metallurgy technology $[2,3]$ because of its advantage such as the low temperature process which allows to apply polymer masks in photolithography. Implantation is a kind of technology which allows introducing non-soluble elements into the material [4]. The process of irradiation is uniform in surface. It is easy to control the concentration of dopants introduced into depth. Thanks to the use of masks of various width, the concentration of dopants may be changed in various areas of surface in one cycle of implantation. The implantation process is well modeled with Monte-Carrlo simulation. Popular software for such modeling is SRIM code [5]. The experimental results agree very well with model results for $\mathrm{Si}$, AIII-BV semiconductors as well as for metals. However, the parameters of implanted CdTe crystals differ considerably from those pointed by SRIM simulation. Therefore, the implantation of CdTE is less applicable in microelectronic technology of devices based on CdTe material. In this paper we present the problems encountered with ion implantation of CdTe. In Section 2 we present preparation of samples for implantation. The physical principles of implantation are discussed in Section 3 . The post implanted annealing of the samples is analysed in this section as well. In Section 4 the classical problems with implantation of $\mathrm{B}$ an As into CdTe in the aim of $p-n$ junction formation are outlined. Structure damage of the samples after implantation is addressed in Section 5 basing mainly on the excellent paper of Rischau et al. [6].

Affiliations of authors should be typed in 9-point Times. They should be preceded by a numerical superscript corresponding to the same superscript after the name of the author concerned. Please ensure that affiliations are as full and complete as possible and include the country.

\section{Preimplantation sample preparation}

The majority of the samples were prepared in the form of platelets of a size of $5 \times 4 \times 1 \mathrm{~mm}^{3}$. Platelets were mechanically polished and chemically etched with a solution of bromine in methanol. The preimplantation annealing of Te rich CdTe in Cd vapour was used for 36 $\mathrm{h}$ at $500^{\circ} \mathrm{C}$ ensuring $\mathrm{Cd}$ overpressure of 0.5 Torr [7].

The implantation with $\mathrm{As}^{+}$at room temperature is not efficient while the damage of structure is so great that the sample becomes semi insulating. The recovery of crystal structure of the crystal structure was not achieved by post implanted annealing within two days at $650^{\circ} \mathrm{C}$ 
[8]. Therefore, the hot temperature implantation had to be applied. For this, the obstacle of high vapour pressure of CdTe should be removed by encapsulating of the samples. It was done by means of $\mathrm{SiO}_{2}$ layers .The layers with $150 \mathrm{~nm}$ are proper for $\mathrm{As}^{+}$ions implantation . $\mathrm{SiO}_{2}$ encapsulating layers are stable for temperatures not exceeding $525^{\circ} \mathrm{C}[8]$.

\section{Implantation overview, implantation parameters and instrumentation}

The main implantation parameters are: temperature of target (T), energy of ions (E), ion current density (I), time of implantation ( $\mathrm{t}$ ), total dose (TD).

The typical set up for ion implantation consists of (Fig.1) source of ions, magnet for ion selection, accelerator, scanning electrostatic plates, beam diaphragm, The environment for all these parts/ factors is high vacuum $\left(10^{-7}\right.$ Torr $)$.

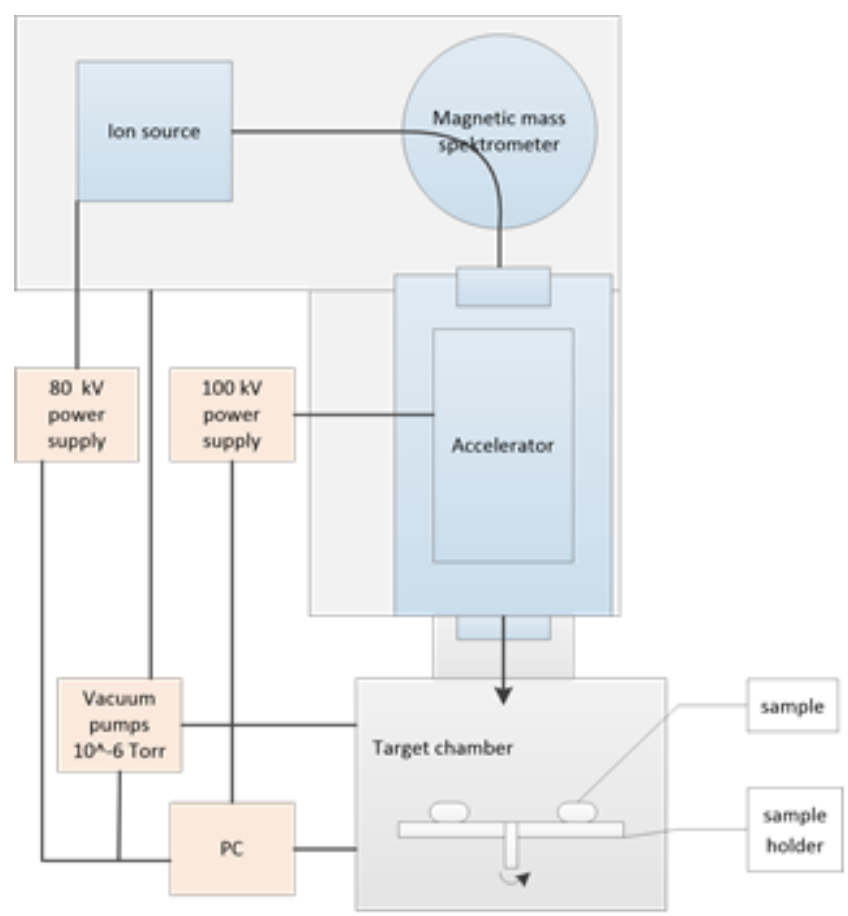

Fig. 1. Scheme of set up for ion implantation.

Ion current density is measured using Faraday box as:

$$
j=I / q e A,
$$

where $A$ is the area of Faraday box, $\mathrm{q}$ is the iconicity number of ions (usually $q=1$ ), e is the elementary charge. The typical currents in ion implantation are $1 \mu \mathrm{A}$ $-10 \mathrm{~mA}$.

The fluency (dose) is defined as

$$
\Phi=j \cdot t
$$

where $t$ is the implantation time.
A dose gives a number of ions per unit area in whole depths of samples. It is not with simple relation to concentration of dopants ( the number of atoms per unit volume while the concentration depends strongly on the depth although laterally it is constant.

Ions with high energy ( usually $50-750 \mathrm{keV}$ ) penetrates the near surface layer ( up to $1 \mu \mathrm{m}$ for high energy). The ions undergo collisions with nucleons or electron of atoms of the target. These collisions are cascade and ions gradually lose the energy. Rate of energy loss into dept $\mathrm{dE} / \mathrm{dx}$ depends on energy of ions and material. The dependence of the rate of energy loss for collision with electrons increases linearly versus $\mathrm{E}^{1 / 2}$. Contrary to this, such dependence for collisions with nucleons is nonlinear showing clear maximum at energy in small range of energy $E$ as it is seen in Fig. 2a . In this range of energy the nuclear loss of energy is dominated whereas for high energy ions the electronic loss is prevailing. In Fig. $2 a$ and $2 b$ Such rate of energy loss for CdTe crystals implanted with $\mathrm{Cr}, \mathrm{B}$, and As were calculated using SRIM code [5] and the results are demonstrated in Fig.2b and Fig.2c.

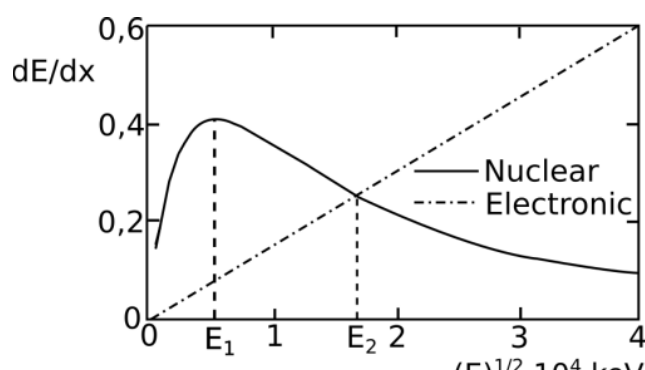

a)

$(\mathrm{E})^{1 / 2} \cdot 10^{4} \mathrm{keV}$

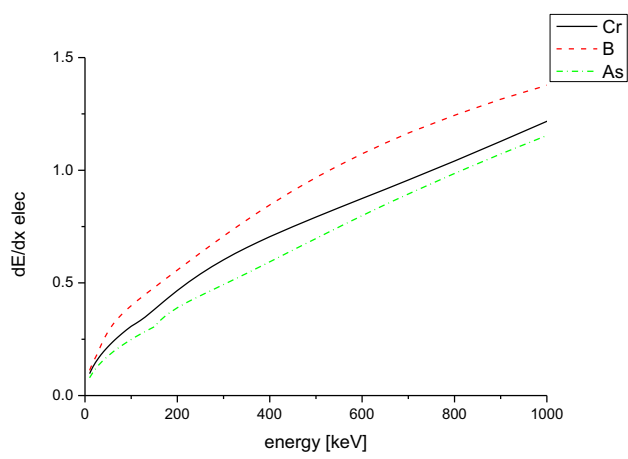

b)

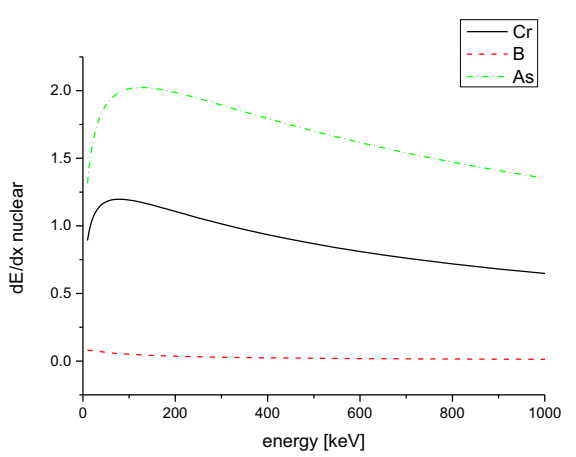

c)

Fig. 2. Dependence of the rate of energy loss on $E^{1 / 2}$ (a), electronic (b) and nuclear (c) rate of energy loss for CdTe implanted with $\mathrm{Cr}, \mathrm{B}$ and As (results of SRIM simulations). 
Irradiation of a solid with high energy ions leads to numerous crystal defect formations on the surface as well in the near surface layer [9-12] :

1) surface defects are: sputtering, roughening, swelling, surface nanopatterning (see[13] and ref. therein),

2) in the bulk the following defects are created: vacancies, interstitials, pattern formation, void formation, recombination, diffusion, sponge structure formation.

In the aim to remove these defects or part of them the annealing after implantation is applied. However, the annealing may create new defects like nucleation of existing defects, nanocluster formation, voids formation ( by nucleation of vacancies ) etc. [14-16].

The implantation of a small area on the surface of the target results in formation of bulk distribution of dopants in $\mathrm{x}, \mathrm{y}, \mathrm{z}$ space in the form of a ball (Fig .3a).

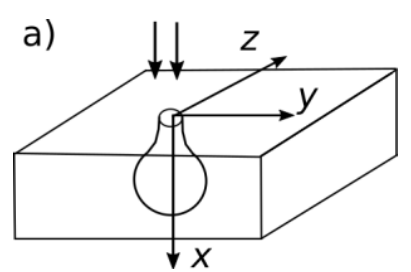

b)
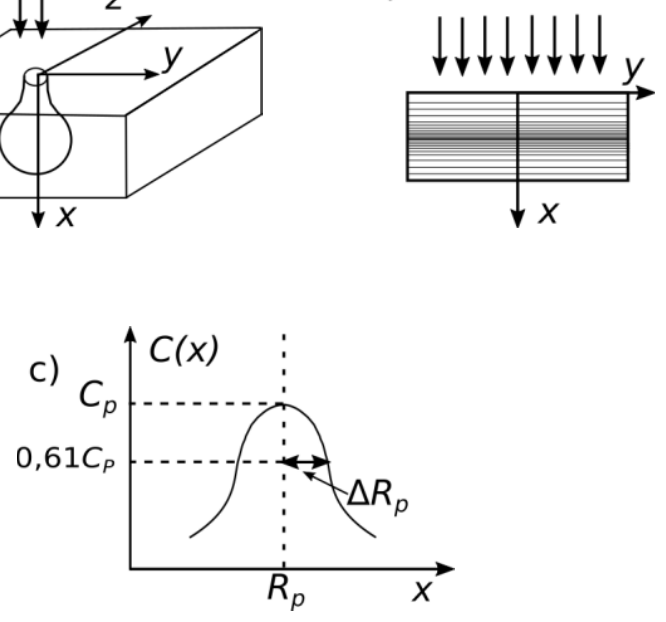

Fig. 3. Scheme of concentration distribution of defects ( or dopants) in the sample implanted by stable ion beam (a), implanted by scanning ion beam (b), one-dimensional Gaussian approximation of defects distribution (c).

Usually the target is scanned by ion-beam, therefore the superposition of implantation at all the lateral position on a surface results in one-dimensional depth profile of dopant ( Fig . 3b ). Such profile can be approximated by Gaussian function (Fig. 3c):

$$
C(x)=C_{p} \exp \left[-\left(x-R_{p}\right)^{2} / 2\left(\Delta R_{p}\right)^{2}\right],
$$

where $R_{p}$ is a projected range, and $\Delta R_{p}$ is a longitudinal straggle.

The parameters $R_{p}$ and $\Delta R_{p}$ depend in a near linear way on graphs $\log R_{p}$ versus $\log E$ or $\log \Delta R_{p}$ versus $\log E$ almost for all the elements implanted.

There are two aims of the post implanted annealing:

1) Restoring the crystallinity of the sample,

2) Introducing dopants into substitute sites of the crystal lattice in order to activate them( electrically or opticaly ). Three types of post implanted annealing are applied:

1) Steady annealing (e. g. $\mathrm{T}=650^{\circ} \mathrm{C}$, in time 1 hour for CdTe).

2) Rapid thermal annealing [17] using the xenon lamps ( heating up to $1000^{\circ} \mathrm{C}$ with velocity more then $50^{\circ} \mathrm{C} / \mathrm{s}$ ).
3) Pulsed laser annealing with nanosecond length of pulses (see [18] and ref. therein).

\section{The p-n junction formation in CdTe crystals}

In the paper of Donnelly at al. [8] the following parameters were established with the aim of formation of type conversion of $\mathrm{n}-\mathrm{CdTe}$ into $\mathrm{p}-\mathrm{CdTe}$ by $\mathrm{As}^{+}$ implantation : $\mathrm{T}=500^{\circ} \mathrm{C}, \mathrm{E}=400 \mathrm{keV}, \mathrm{I}=0.5 \mu \mathrm{A} / \mathrm{cm}^{2}$, $\mathrm{t}=15 \mathrm{~min}$, dose $=3 \cdot 10^{15} / \mathrm{cm}^{2}$. The $\mathrm{p}-\mathrm{n}$ junction formation was supported by U-I characteristics which show forward resistance of $30 \Omega$ and reverse breakdown of $40 \mathrm{~V}$.

Chu [7] implanted n-type undopted CdTe by $\mathrm{As}^{+}$ions with energy $60 \mathrm{keV}$, and dose $10^{15}$. Electrical and photovoltaic properties were measured. Density of holes as a function of distance from implanted surface were determined by Hall measurements after periodic successive etching of the sample. From these measurements it follows that the effects of the implantation were observed at depts grater then $250 \mathrm{~nm}$, whereas the modeling projected range for $\mathrm{As}^{+}$ions at such energy is about $29 \pm 17 \mathrm{~nm}$.

Further continuation of implantation of CdTe crystals in order to obtain $\mathrm{p}-\mathrm{n}^{+}$junction was ensured for $\mathrm{p}$-type $\mathrm{Cd}_{\mathrm{x}} \mathrm{Hg}_{1-\mathrm{x}} \mathrm{Te}[19,20]$. The $\mathrm{n}$ - type conductivity may be produced by implantation of $\mathrm{B}$ [21], $\mathrm{Al}$ [22], In [23 ], $\mathrm{Zn} \mathrm{[23} \mathrm{],} \mathrm{Hg} \mathrm{[24]} \mathrm{as} \mathrm{well} \mathrm{as} \mathrm{Au} \mathrm{and} \mathrm{P} \mathrm{(see} \mathrm{Capper} \mathrm{[1]}$ p.160). The electrical properties of ion implanted layers in $\mathrm{Cd}_{\mathrm{x}} \mathrm{Hg}_{1-\mathrm{x}} \mathrm{Te}$ were investigated by Margalit et al. [20]. Dopants ( B, Ar, In, P, Au ) were implanted at $150 \mathrm{keV}$, with dose $10^{11}-10^{15} 1 / \mathrm{cm}^{2}$. The samples were annealed at temperature $150^{\circ} \mathrm{C}$ during $24 \mathrm{~h}$ in vacuum The samples were encapsulated by $200 \mathrm{~nm}$ thick CdTe layer. The results demonstrate that the crystal structure damage rather then kind of dopants converts p-type into n-type conductivity. The authors proposed to applicate the implantation for formation of accumulated surface for surface passivation and for formation of Ohmic contacts on n-type material.

Recently, ion implantation of $\mathrm{CdHgTe}$ is well known method for fabrication infrared photovoltaic devices. The paper [25] presents a short review on technology of production of $\mathrm{CdHgTe}$ fotodiodes by implantation As into n-type matrix.

\section{5 lon - beam induced damage in CdTe}

Implantation damage in CdTe implanted with isotope of host ions ${ }^{111} \mathrm{Cd}$ at $60 \mathrm{keV}$ at room temperature were investigated by Achtziger et al. [26]. Dose was low: $2 \cdot 10^{11} 1 / \mathrm{cm}^{2}-6 \cdot 10^{11} 1 / \mathrm{cm}^{2}$ The host atoms were choosen for implantation in the aim to avoid any chemical activity of implants. Annealing treatment was carried out for several temperatures in the range $723 \mathrm{~K}-1073 \mathrm{~K}$ Characterization of the implanted and annealed samples was made by perturbed angular spectroscopy and by deep-level transient spectroscopy. For this last technique parallely, the set of nonimplanted samples was annealed and measured providing good reference data. The 
conclusions from these studies are: 1) At low doses all implanted atoms occupy substitutional lattice sites. Local crystal environment of these atoms is almost perfect before annealing:; after annealing at $673 \mathrm{~K}$ it is perfect, 2) After implantation no additional electrically active centers have been observed.

C. W. Rischau et al. [6] have implanted CdTe single crystals with orientation (111) and (112). As dopants two types of ions were chosen: the light ions $\mathrm{Ar}^{+}$and the heavy ions $\mathrm{Sb}^{++}$having mass comparable with masses of self $\mathrm{Cd}$ and $\mathrm{Te}$ atoms. The implantation was carried out in two temperatures: $5 \mathrm{~K}$ and in room temperature. These two implantation temperatures should help to decide which mechanism of implanted defects formation, thermal or non-thermal is responsible for an unusually small, but deep damage of structure in CdTe. The energy of ions was $270 \mathrm{keV}$ for $\mathrm{Ar}^{+}$and $730 \mathrm{keV}$ for $\mathrm{Sb}^{++}$. Such values of energy were chosen to obtain the projected range for both ions $R_{p}=220 \mathrm{~nm}$. Such value lies well beyond of the positions of surface atoms $\mathrm{Cd}$ and Te in RBS spectra. The dose was ranging from $10^{11}$ $1 / \mathrm{cm}^{2}$ to $10^{17} 1 / \mathrm{cm}^{2}$. The RBS spectra of samples irradiated with $\mathrm{Ar}^{+}$ions did not differ considerably for both orientations of samples at room temperature. Moreover, at low temperature $(15 \mathrm{~K})$ this spectra are similar to those in room temperature. There is expressive dependence of lattice disorder on the dose. In spite of this, the channeling spectra do not reach random spectrum even for the highest dose $5 \cdot 10^{16} 1 / \mathrm{cm}^{2}$. This indicates that the crystal structure is resistive against amorphization even at a dose of $10^{17} 1 / \mathrm{cm}^{2}$. The defect distribution obtained from RBS spectra show deformed Gaussian profiles with $R_{p}=250 \mathrm{~nm}$ independently on the orientation of crystals at room temperature. The tail of distribution is long and is equal to $z=2 R_{p}$ up to $z=4 R_{p}$. This is a great discrepancy with SRIM results. The defects distribution at $15 \mathrm{~K}$ differs considerably from these latter. At doses higher than $2 \cdot 10^{15}$ the distribution is flat extended up to $4 \mathrm{R}_{\mathrm{p}}$ with the maximum at $350 \mathrm{~nm}$ independent on the orientation and type of ions. There is also significant increase of defects at surface of the sample. This surface damage increases significantly with the dose increase. However, for both temperatures the buried damage layer placed in the dept $100 \mathrm{~nm}$ below the surface and having the width of about $500 \mathrm{~nm}$ is formed, but this layer is not amorphous. Taking these results into consideration, Rischau [6] concluded that the high resistance of the CdTe crystals to amorphization follows not from thermal effects but from high ionicity of the crystal studied. Such conclusion were formulated earlier by Gettings at al. [27] and by Uzan-Saguy et al.[28].

Ion implantation of heavy Xe ions into (111) oriented single crystals of CdTe was studied by Petric at al. in the paper [29]. The disorder was investigated using Rutherford backscattering spectrometry ( RBS) with $\mathrm{He}^{+}$ ions [30]. The simulation was done using the SRIM software [5].The energy of ions was $350 \mathrm{keV}$ and the doses were as follows: $1 \cdot 10^{13} 1 / \mathrm{cm}^{2}, 2 \cdot 10^{13} 1 / \mathrm{cm}^{2}$, $3 \cdot 10^{13} 1 / \mathrm{cm}^{2}, 4 \cdot 10^{13} 1 / \mathrm{cm}^{2}, 6 \cdot 10^{13} 1 / \mathrm{cm}^{2}, 8 \cdot 10^{13} 1 / \mathrm{cm}^{2}$, $12 \cdot 10^{13} 1 / \mathrm{cm}^{2}, 16 \cdot 10^{13} 1 / \mathrm{cm}^{2}$. Parallely, for comparison, an identical implantation was done on Si single crystals. The experimental results from RBS measurements and results from modeling ( SRIM) exhibit a great difference in full width at half maximum (FWHM) of damage profile created by Xe ions. For Si samples the FWHM is $150 \mathrm{~nm}$ whereas for CdTe samples the FWHM is 90 $\mathrm{nm}$. These values are confirmed by RBS analysis. Moreover, RBS spectra made for $\mathrm{Si}$ and $\mathrm{CdTe}$, both implanted with the Xe ions, show the great influence of disorder on the dose in the case of Si sample. For dose $6 \cdot 10^{14} 1 / \mathrm{cm}^{2}$ the channeling spectrum is comparable with the spectrum of random RBS. Contrary to this, in the case of CdTe the channeling spectra for all doses lie near the virgin spectrum which means that the disorder is very weak even for doses exceeding amorphization threshold calculated from the SRIM code ( several $10^{16}$ $1 / \mathrm{cm}^{2}$ ). From the tails of RBS spectra follows that the extended defects, mainly dislocation loops, prevail in this material after implantation. This seems to be in conformity with results presented in papers of Leo et al. [31-33] where the formation of dislocation loops was explained by high mobility of interstitial $\mathrm{Te}$ and enhanced recombination of defects in the depth of the sample. On the other hand, the defects near the surface were grasped by the surface and therefore the near surface layer was protected again amorphization even for high doses.

\section{CONCLUSIONS}

Usually, the samples are encapsulated before implantation. It seems that this rule should be obligatory in the case of CdTe implantation with high energy. However, this problem is omitted in the works cited. The high stability of the crystal structure seems to promise auspicious future of this material. Its application in doping of new elements allows to obtain new properties of this material. Heavy doping with magnetic ions seems to be one of these prospective new application of ion implantation of CdTe crystals or layers.

\section{Acknowledgments}

The support from the Center for Innovation and Transfer of Natural Science and Engineering Knowledge at the University of Rzeszow is gratefully acknowledged.

\section{References}

1. P. Capper, Properties of Narrow-Gap CadmiumBased Compounds, (United Kingdom 1994)

2. J. F. Ziegler (ed.) Ion Implantation (Science and Technology, Academic Press 1988)

3. E. Rimini, Ion Implantation, Basic to Device Fabrication, (Kluver Academic Publ., Boston 1995)

4. O. Meyer, A. Turos, Materials Science Reports 2, 7, 373-468 (1987)

5. The Stopping and Range of Ions in Matter, http://www.srim.org.

6. C.W. Rischau, C.S. Schnohr, E. Wendler, W. Wesch,J. Appl. Phys. 109, 113531 (2011) 
7. M. Chu, A.L. Fahrenbruch, R.H. Bube, J.F. Gibbons, J. Appl. Phys. 49, 322 (1978)

8. J.P. Donnelly, A.G. Foyt, E.D. Hinkley, W.T. Lindley, J.O. Dimmock, Appl. Phys. Lett. 12, 303 (1968)

9. R. Böttger, K.H. Heinig, L. Bischoff, B. Liedke, R. Hübner, W. Pilz, Phys. Status Solidi - Rapid Res. Lett. 7, 501 (2013)

10. R.A. Wilhelm, E. Gruber, R. Ritter, R. Heller, S. Facsko, F. Aumayr, Phys. Rev. Lett. 112, 153201 (2014)

11. R. Böttger, K.H. Heinig, L. Bischoff, B. Liedke, S. Facsko, Appl. Phys. A Mater. Sci. Process. 113, 59 (2013)

12. R. Ritter, R.A. Wilhelm, M. Stöger-Pollach, R. Heller, A. Mücklich, U. Werner, et al., Appl. Phys. Lett. 102, 063112 (2013)

13. O. El-Atwani, S.A. Norris, K. Ludwig, S. Gonderman, J.P. Allain, Sci. Rep. 5, 18207 (2015) 18207

14. S. Zhou, K. Potzger, G. Zhang, A. Mücklich, F. Eichhorn, N. Schell, et al., Phys. Rev.B 75, 085203 (2007)

15. S. Zhou, K. Potzger, J. Von Borany, R. Grötzschel, W. Skorupa, M. Helm, et al., Phys. Rev. B 77, 035209 (2008)

16. S. Zhou, A. Shalimov, K. Potzger, N.M. Jeutter, C. Baehtz, M. Helm, et al., Appl. Phys. Lett. 95, 192505 (2009)

17. T.E. Seidel, D.J. Lischner, C.S. Pai, R.V. Knoell, D.M. Maher, D.C. Jacobson, Nuclear Instruments and Methods in Physics Research Section B: Beam Interactions with Materials and Atoms, 7-8, Part 1, 251-260 ( 1985)

18. S. Zhou, Dilute ferromagnetic semiconductors prepared by the combination of ion implantation with pulse laser melting (Topic Review), J. Phys. D: Appl. Phys. 48, 263001 (2015).

19. C. Uzan-Saguy, J. Vac. Sci. Technol. A Vacuum, Surfaces, Film. 10, 3246 (1992)

20. S. Margalit, Y. Nemirovsky, I. Rotstein, J. Appl. Phys. 50, 6386 (1979)

21. M. Lanir, A.H.B. Vanderwyck, and C.C. Wang, J. Appl. Phys. 49, 6182 (1978)

22. J. Marine and C. Motte, Appl. Phys. Lett. 23, 450 (1973)

23. E. Igras, J. Piotrowski, and I.Z. Higersberger, Electron Technol. 10,63 (1977)

24. G. Fiorito, G. Gasparrini, and F. Svelto, Appl. Phys. Lett. 17, 105 (1978)

25. L. Mollard, G. Destefanis, N. Baier, J. Rothman, P. Ballet, J.P. Zanatta, et al., J. Electron. Mater. 38,1805 (2009)

26. N Achtziger, J Bollmann, Th Licht, B. Reinhold, U Reislöhner, J Röhrich, M Rüb, M Wienecke, W Witthuhn, Semiconductor Science and Technology 11, 947 (1996)
27. M.Gettings, K.G. Stephens, Radiat. Eff. 22, 53 (1974)

28. C. Uzan-Saguy, D. Comedi, V. Richter, R. Kalish, and R. Triboulet, J. Vac. Sci. Technol. A 7, 2575 (1989)

29. P. Petrik, N.Q. Khánh, J. Li, J. Chen, R.W. Collins, M. Fried, et al., Phys. Status Solidi Curr. Top. Solid State Phys. 5, 1358 (2008)

30. L.C. Feldman, J.W. Mayer, S.T. Picraux, Materials Analysis by Ion Channeling, (Academic Press 1982)

31. G. Leo, A. Traverse, M. O. Ruault, and A. V. Drigo, Nucl. Instrum. Methods Phys. Res. B 63, 41 (1992)

32. G. Leo and M. O. Ruault, J. Appl. Phys. 73, 2234 (1993)

33. G. Leo, A. V. Drigo, and A. Traverse, Mater. Sci. Eng. B 16, 123 (1993) 